\title{
A VUELTAS CON LA NOCIÓN DE SOBERANÍA: REFLEXIONES SOBRE LA CONTRIBUCIÓN DE ERNESTO GARZÓN VALDÉS
}

\author{
Isabel Turégano Mansilla \\ Universidad de Castilla-La Mancha
}

RESUMEN. El concepto de soberanía, teóricamente independizado de los problemas de fundamentación política, sirvió en la teoría jurídica como instrumento adecuado para explicar el derecho al margen de una noción normativa de validez. La conocida distinción de Garzón Valdés entre un soberano-0 y un soberano- 1 constituye una herramienta conceptual necesaria para acomodar tal análisis jurídico a la realidad del Estado constitucional. Sin embargo, como también Garzón Valdés ha tenido ocasión de expresar, la doctrina de la soberanía se halla impregnada de importantes connotaciones normativas que terminan frustrando la pretensión de neutralidad con que fue empleada. Ocurre así cuando la propia teoría se cuestiona las bases del fenómeno social en que consiste el poder o cuando trata de adaptarse a los Estados constitucionales transformándose en poder constituyente.

Palabras clave: Ernesto Garzón Valdés, soberanía, poder constituyente.

ABSTRACT. The concept of sovereignty, theoretically made independent of problems of political justification, was used by legal theory as an appropriate instrument to explain law aside a normative notion of validity. The well-known Garzón Valdés's distinction between a sovereign-0 and a sovereign-1 is a necessary conceptual tool to reconcile such legal analysis to the reality of a constitutional State. However, as Garzón Valdés has also had the opportunity to express, the doctrine of sovereignty has significant normative connotations that end up by frustrating the aim of neutrality with which it was used. Such is the case when the theory questions the basis of the social phenomenon power consists of or when it tries to fit to constitutional States transforming itself into constituent power.

Keywords: Ernesto Garzón Valdés, sovereignty, constituent power. 


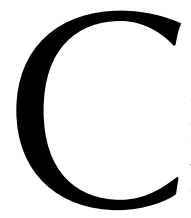

omo ya señaló el propio GARZÓn VALDÉs en el célebre primer volumen de esta publicación, la noción de soberanía es una de esas cuestiones que HART denominara «recurrentes» que reaparecen de cuando en cuando en los escritos de teoría jurídica y que, al mismo tiempo, no deja de ocupar un lugar destacado en el discurso moral en el que desembocan muchas de las cuestiones filosófico-jurídicas.

En la teoría jurídica positivista la doctrina de la soberanía se empleó en muchos casos como instrumento adecuado para dar cuenta del derecho como conjunto de hechos sociales complejos que pueden ser identificados sin asumir compromiso valorativo alguno (validez como existencia de instituciones sociales y no como razones que justifican acciones). El concepto de soberano reflejaría la realidad social del poder o dominio sobre la base de una actitud general y habitual de obediencia sin plantearse la cuestión del fundamento normativo del mismo. Como señaló GARZÓN VALDÉs, esta doctrina lleva a presentar la base del ordenamiento jurídico a partir de actos de voluntad que dictan las normas jurídicas supremas, para lo que es suficiente asumir un concepto neutro de soberano como origen de la cadena de validez normativa.

El carácter independiente o no subordinado que completa la definición de la soberanía implica que no es posible limitar jurídicamente al soberano, puesto que un soberano sometido a límites jurídicos estaría sujeto a un soberano superior y ello resulta contradictorio: sería soberano y no soberano a un tiempo. Esta conclusión podría resultar desmentida por la propia realidad del Estado constitucional de Derecho en cuya norma fundamental suelen incorporarse disposiciones de reforma constitucional que limitan formal y materialmente la competencia de la autoridad jurídica suprema. Al conocido debate acerca de la posibilidad o no de reformar válidamente tales disposiciones de reforma subyace la búsqueda de un instrumento conceptual para dar cuenta de la vinculación de la autoridad normativa última. Así los argumentos de autorreferencia y de contradicción de Alf Ross (Ross, 1953: 79-81; 1969: 1-24); la analogía con situaciones de precompromiso personal asimilando una imposibilidad fáctica a una imposibilidad deóntica; o la interpretación de los límites a la reforma constitucional en términos del punto de vista interno hartiano de Hartmut KliEmt (KLIEMT, 1978: 99-108).

Los problemas de cada una de estas argumentaciones no hacen si no mostrar que los límites a la autoridad suprema no pueden ser jurídicos y que, en ningún caso, puede hablarse de validez en relación con sus actos. Cuando la constitución es reformada por un órgano o mediante un procedimiento distintos de los establecidos en la disposición de reforma constitucional o más allá de los límites materiales que ésta determina, se produce una norma inválida - en el sentido de que su promulgación no ha sido autorizada - que puede iniciar una nueva cadena de validez en caso de que la reforma resulte eficaz. Conforme a la definición de VON WRIGHT, el agente que realiza ese acto normativo que no es contenido de otra norma superior actúa como soberano o autoridad suprema (WRIGHT, 1963: 192). Pero ello no obsta para que pueda pensarse en una autoridad jurídica última con competencias limitadas. Y este es el sentido

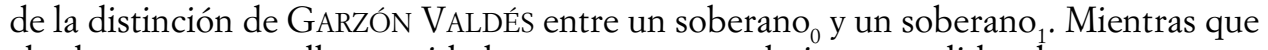
el soberano ${ }_{0}$ es aquella autoridad cuyas normas no derivan su validez de otras normas del sistema, el soberano ${ }_{1}$ es aquella a la que las normas del sistema atribuyen en última instancia la competencia para regular jurídicamente la sociedad. Sólo respecto de 
los actos normativos de este soberano ${ }_{1}$ puede predicarse validez o invalidez, competencia o incompetencia. Pero para dar cuenta de esta idea de una autoridad jurídica vinculada hay que presuponer conceptualmente la de soberano ${ }_{0}$. Sin soberano ${ }_{0}$ no exis-

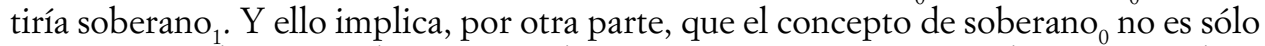
un concepto de contenido ético o político sino un concepto con relevancia jurídica. GARZÓN VALDÉS afirma que «[n]o tendría mucho sentido hablar de soberano ${ }_{0}$ si luego resulta que no hay soberano ${ }_{1}$ ya que la definición de soberano $0_{0}$, tal como aquí es entendida, hace referencia a una cadena de actos normativos entre los que precisamente figuran los que realiza el soberano ${ }_{1}$ en ejercicio de su competencia (suprema o no). En este sentido, el concepto de soberano ${ }_{0}$ no es jurídicamente irrelevante» (GARZÓN VALDÉS, 1983a: 198).

La anterior construcción emplea la noción de soberano, como he señalado al comienzo, para explicar el Derecho al margen de una noción normativa de validez. Sin embargo, lo cierto es que en gran parte de los escritos que han dedicado su atención al problema de la soberanía se cuelan o irrumpen abiertamente planteamientos normativistas y termina siendo difícil conciliar la doctrina de la soberanía con una teoría del Derecho reducida a la tesis de las fuentes sociales. Ocurre así, en primer lugar, cuando la teoría de la soberanía, concebida inicialmente como concepto para el análisis de lo jurídico, comienza a cuestionarse las bases del fenómeno social en que consiste el poder o dominio. Frente a la imagen reduccionista del poder en términos de regularidades externas de comportamiento, un concepto más complejo del poder social ofrece un panorama distinto de las relaciones entre éste y el Derecho. Conforme al mismo, el fenómeno del poder no se reduce a un hecho simple ajustable al esquema de mandatoobediencia sino que se concibe como práctica social compleja que se articula mediante normas sociales. Tales prácticas sociales de naturaleza normativa incorporan, entre otros, los criterios de identificación de la agencia a la que se confiere la potestad de regular jurídicamente la interacción social. De este modo, la identificación del soberano responde a un reconocimiento social previo de la necesidad del Derecho como guía de la conducta y la aceptación y aprobación de una autoridad normativa (vid. LAPORTA, 1996: 445-447). Éste es el sentido de la moralidad positiva de John Austin, que, al requerir y especificar los caracteres de quienes van a detentar la soberanía, se puede concebir como el fundamento no sólo de la existencia sino también de la normatividad del Derecho. El soberano de AUSTIN no es el poder que logra imponer sus mandatos por la fuerza, sino aquel o aquellos a quienes la sociedad reconoce la capacidad de crear una estructura normativa que haga posible la coexistencia. Esta idea, que aparece ya apuntada en algunos fragmentos de sus lecciones (Austin, 1861-1863: 116-117, 321, 746), se hace evidente en su último ensayo, $A$ Plea for the Constitution, donde afirma que la supervivencia de un determinado orden jurídico depende de un sentimiento compartido por la opinión pública de vinculación a la forma de gobierno establecida y de reconocimiento de su necesidad para mantener la estabilidad social (AUSTIN, 1859; TURÉGANO, 2001: 416-422).

Además, en segundo lugar, el contenido normativo del concepto de soberano se evidencia en mayor medida cuando la teoría imperativista se trata de adaptar a los Estados constitucionales. La conversión del soberano en poder constituyente o legislador originario termina planteando más problemas que los que puede resolver. Empleado 
con una función explicativa, el concepto de poder constituyente sirve efectivamente para poner de manifiesto que la limitación de la autoridad jurídica suprema presupone lógicamente un poder extrajurídico que le autoriza y delimita su ámbito de actuación. Éste creo que es el sentido de la distinción entre soberano y soberano $_{1}$. Sin embargo, como ya señalaran CARRIÓ y NINO, resulta problemática la aplicación de un término como el de «poder» propio de contextos normativos en una sede en la que por definición las normas están ausentes, puesto que puede servir como legitimación encubierta del mero hecho del ejercicio efectivo de la fuerza (CARRIÓ, 1973; NINO, 1985). Evitar tal equívoco implica reconocer que el uso del concepto de poder constituyente originario depende de consideraciones ético-normativas, como afirmó GARZÓN VALDÉS en su escrito comparativo del pensamiento de John Austin y Hermann HelleR, en el que hace suyas las palabras de este último para quien «existencia y normatividad del poder constituyente no están en oposición sino que se condicionan recíprocamente» (GARZÓN VALDÉS, 1983b: 180).

Es sólo en este sentido ético-normativo en el que el concepto de poder constituyente cobra su sentido en el constitucionalismo moderno. En éste su naturaleza voluntarista queda delimitada por su función garantista: el poder constituyente no es una posibilidad absoluta de crear o modificar en todo momento una organización político-jurídica sino instrumento de realización de un modelo concreto de legitimación política y justicia jurídica. En este sentido, el concepto hace referencia a un espacio que no debe ser llenado por la voluntad político-jurídica, a un vacío de poder que no ha de ser ocupado por ninguna facción política. Por ello, se habla del necesario agotamiento del poder constituyente en el Estado constitucional, una vez que los valores y principios fundamentales abandonan la esfera de la trascendencia y se incorporan a las constituciones (DOGLIANI, 1996; LuCIANI, 1996; VeGA, 1985).

Es en el marco de estas premisas garantistas donde adquiere un sentido justificatorio la distinción entre un constituyente originario y uno derivado. La afirmación de la continuidad del poder constituyente en el orden jurídico bajo la forma de un poder ilimitado de reforma tiene una connotación negativa de restricción de las garantías constitucionalmente reconocidas.

Tal tesis, sin embargo, se considera discutible desde la perspectiva de quienes priman el valor de la democracia sobre el de los derechos y afirman que, puesto que los derechos se incorporan a la constitución mediante un acto de voluntad contingente, la posibilidad de su revisión se convierte en una necesidad ineludible. Desde esta perspectiva, el problema de la fundamentación de los derechos no se limita a analizar su sustrato normativo sino también a determinar cómo insertar los derechos ya fundamentados en las ordenaciones vigentes (PINTORE, 2001: 244-246). A partir de la premisa del desacuerdo moral, no ya acerca de la existencia de verdades morales objetivas, sino - aun entre quienes desean evitar el relativismo- acerca del modo de acceder a ellas, se exige el diseño de procedimientos continuos para la toma de decisiones colectivas (WALDRON, 1993: 32; 1999: 176-181). De este modo, la regulación de la revisión constitucional es la que crea las condiciones necesarias para debatir colectivamente las cuestiones constitucionales. El concepto de poder constituyente se introduce, así, en el marco jurídico como posibilidad constante de replantear el pacto constitucional (vid. PALOMBELla, 2000). El riesgo de tal concepción es que la prioridad absoluta que con- 
fiere a la soberanía popular sobre cualquier otro criterio normativo puede llevar a justificar los actos de toma de decisiones constitucionales por sí mismos con independencia de principios sustantivos independientes.

\section{BIBLIOGRAFÍA CITADA}

Austin, J., 1859: A Plea for the Constitution, London: John Murray.

- 1861-1863: Lectures on Jurisprudence or the Philosophy of Positive Law, 5. ${ }^{\text {ed. }}$ revisada y editada por CAMPBELL, R., London: John Murray, 1885.

CARrió, G. R., 1973: «Sobre los límites del lenguaje normativo», Notas sobre derecho y lenguaje, Buenos Aires: Abeledo-Perrot, 1990.

De VeGA, P., 1985: La reforma constitucional y la problemática del poder constituyente, Madrid: Tecnos.

Dogliani, M., 1996: «Potere costituente e revisione costituzionale nella lotta per la costituzione», Zagrebelsky, G.; Portinaro, P. P., y Luther, J. (eds.), Il futuro della costituzione, Torino: Einaudi, pp. 270-277.

GARZÓN VALDÉS, E., 1983a: «Las limitaciones jurídicas del soberano», Derecho, ética y política, Madrid: Centro de Estudios Constitucionales, 1993: pp. 181-200.

— 1983b: «Hermann Heller y John Austin: Un intento de comparación», Derecho, ética y politica, cit.: pp. 157-180.

Kliemt, H., 1978: «Can There Be Any Constitucional Limits to Constitutional Powers?», Munich Social Science Review, 4: pp. 99-108.

LAPORTA, F., 1996: «Poder y Derecho», GARZÓn VALDÉs, E., y LAPORTA, F. (eds.), El Derecho y la justicia, Enciclopedia Iberoamericana de Filosofía, Madrid: Trotta-CSIC-BOE, pp. 441453.

LuCiAni, M., 1996: «L'antisovrano e la crisi delle costituzioni», Revista de Diritto Costituzionale, 1: pp. 147-153.

NINO, C. S., 1985: «La competencia del constituyente originario y el carácter moral de la justificación jurídica», La validez del Derecho, Buenos Aires: Astrea, pp. 41-68.

Palombella, G., 2000: Constitución y soberanía. El sentido de la democracia constitucional, trad. y prólogo de CALVO GONZÁlEZ, J., Granada: Comares.

Pintore, A., 2001: «Derechos insaciables», FERRAJOli, L.: Los fundamentos de los derechos fundamentales, editado por DE CABO, A., y PIsarello, G., Madrid: Trotta.

Ross, A., 1953: Sobre el Derecho y la justicia, trad. de CARRIÓ, G. R., Buenos Aires: Eudeba, 1963.

— 1969: «On Self-Reference and a Puzzle in Constitutional Law», Mind, 78: pp. 1-24.

Turégano, I., 2001: Derecho y moral en John Austin, Madrid: Centro de Estudios Políticos y Constitucionales.

VON Wright, G. H., 1963: Norma y acción. Una investigación lógica, Madrid: Tecnos.

WALDRON, J., 1993: «A Right-Based Critique of Constitutional Rights», Oxford Journal of Legal Studies, 13.

- 1999: Law and Disagreement, Oxford: Oxford University Press. 